\title{
¿Mejora el PILATES la salud mental?
}

\author{
*Julio Torales ${ }^{1}$, Marcos Almirón ${ }^{1}$, Israel González ${ }^{1}$, Rodrigo Navarro ${ }^{1}$, \\ Marcelo O'Higgins ${ }^{1}$, João Castaldelli-Maia ${ }^{2}$, Antonio Ventriglio ${ }^{3}$ \\ ${ }^{1}$ Universidad Nacional de Asunción, Facultad de Ciencias Médicas. Paraguay \\ ${ }^{2}$ Universidad de São Paulo, Instituto de Psiquiatría. Brasil \\ ${ }^{3}$ Universidad de Foggia, Departamento de Medicina Clínica y Experimental. Italia
}

Cómo referenciar este artículo/ How to reference this article:
Torales J, Almirón M, González I, Navarro R, O’Higgins M, Castaldelli-Maia J, et al. ¿Mejora el PILATES la salud mental?. Mem. Inst. Investig. Cienc. Salud. 2019; 17(3): 97-102

\section{RES U M E N}

El Pilates es una forma alternativa de terapia física de baja a moderada intensidad, predominantemente realizada en el suelo y que se enfoca en abordar estabilidad, fuerza muscular, flexibilidad, respiración y postura. Existe evidencia que sostiene su utilidad en la salud física. En cambio, la evidencia es todavía limitada en cuanto a su efectividad para mejorar la salud mental. El objetivo de este artículo de revisión fue resaltar la evidencia preliminar que apoya la utilidad del Pilates en la mejora de la salud mental y variables psicológicas de las personas. Esta fue una revisión narrativa, no sistemática, centrada en literatura primaria, seleccionada de una búsqueda en las bases de datos PubMed y SciELO. Los términos clave que se utilizaron fueron los siguientes: "Pilates y salud mental", "Pilates y trastornos mentales" y "Pilates y psiquiatría". Los resultados fueron complementados con literatura secundaria (libros y capítulos de libros). Todos los miembros del equipo de investigación participaron en la revisión de la literatura. Todo lo anterior se realizó a fin de brindar una orientación general que sirva a médicos psiquiatras y a fisioterapeutas en el diseño de planes terapéuticos que complementen a los psicofármacos, la psicoterapia y al ejercicio físico convencional.

Palabras clave: Pilates, salud mental, variables psicológicas.

\section{Does PILATES improve mental health?}

\begin{abstract}
A B S T R A C T
Pilates is an alternative form of physical therapy of low-to-moderate intensity, predominantly performed on the floor and which focuses on addressing stability, muscle strength, flexibility, breathing and posture. There is evidence supporting its usefulness in physical health. In contrast, the evidence is still limited in terms of its effectiveness in improving mental health. The objective of this review article was to highlight the preliminary evidence that supports the usefulness of Pilates in the improvement of mental health and psychological variables of people. This was a non-systematic narrative review, focused on primary literature, selected from a search in PubMed and SciELO databases. The key terms used were: "Pilates and mental health", "Pilates and mental disorders", and "Pilates and psychiatry". The results were supplemented with secondary literature (books and book chapters). The research was carried out in order to provide a general orientation that would serve psychiatrists and physiotherapists in the design of therapeutic plans that complement psychopharmaceuticals, psychotherapy and conventional physical exercise.
\end{abstract}

Keywords: Pilates, mental health, psychological variables.

Fecha de recepción: abril 2019. Fecha de aceptación: julio 2019

Autor correspondiente: Julio Torales. Cátedra de Psiquiatría (Carrera de Medicina) y Cátedra de Metodología de la Investigación (Carrera de Kinesiología y Fisioterapia), Facultad de Ciencias Médicas, Universidad Nacional de Asunción, Campus Universitario, San Lorenzo, Paraguay.

Email: jtorales@med.una.py 


\section{INTRODUCCIÓN}

Existe evidencia abrumadora que el ejercicio físico es beneficioso para la salud mental en adultos sanos, en pacientes crónicamente enfermos y en aquellos aquejados de sintomatología depresiva o ansiosa ${ }^{(1-7)}$. Asimismo, en estos últimos, un notable número de estudios longitudinales y transversales ha demostrado que el ejercicio físico convencional (como, por ejemplo, el ejercicio aeróbico) se constituye en una estrategia preventiva y en un enfoque adyuvante del tratamiento de los trastornos mentales ${ }^{(8,9)}$. No obstante, todavía existe poca evidencia que apoye el beneficio potencial de formas de ejercicio no convencional.

El Pilates es una forma alternativa de terapia física de baja a moderada intensidad, predominantemente realizado en el suelo y que se enfoca en abordar estabilidad, fuerza muscular, flexibilidad, respiración y postura ${ }^{(10,11)}$. En la salud física, el Pilates ha demostrado efectos positivos en la flexibilidad, equilibrio dinámico y resistencia muscular en poblaciones sanas, y en el manejo del dolor (principalmente de espalda), prevención de caídas y estado físico general en poblaciones de adultos mayores ${ }^{(12,13)}$. En cambio, todavía existe evidencia limitada en cuanto a su utilidad en la salud mental, aunque ha demostrado ser efectivo para mejorar la sintomatología depresiva y el estado de ánimo en adultos mayores y estudiantes, así como la calidad del sueño y calidad de vida en estudiantes, personas de edad media y adultos mayores ${ }^{(14-17)}$.

El objetivo de este artículo de revisión es resaltar la evidencia preliminar que apoya la utilidad del Pilates en la mejora de la salud mental, a fin de brindar una orientación general que sirva a médicos psiquiatras y a fisioterapeutas en el diseño de planes terapéuticos que complementen a los psicofármacos, la psicoterapia y al ejercicio físico convencional.

Esta fue una revisión narrativa, no sistemática, centrada en literatura primaria, seleccionada de una búsqueda en las bases de datos PubMed y SciELO. Los términos clave que se utilizaron fueron los siguientes: "Pilates y salud mental", "Pilates y trastornos mentales" y "Pilates y psiquiatría". Los resultados fueron complementados con literatura secundaria (libros y capítulos de libros). Todos los miembros del equipo de investigación participaron en la revisión de la literatura.

\section{PILATES Y SALUD MENTAL}

La evidencia con respecto a los beneficios que poseen los ejercicios físicos no convencionales, como el Pilates, sobre la salud física está creciendo; no obstante, todavía existe muy poca evidencia disponible acerca de los beneficios de los mismos sobre la salud mental. Algunos investigadores han reportado que un programa de Pilates puede producir mejoras físicas y psicológicas en la salud humana ${ }^{(18,19)}$, mientras que otros han resaltado los beneficios del mismo en la calidad de vida, la salud mental, la fatiga, el estado físico y los tipos corporales ${ }^{(20-25)}$.

El Pilates actualmente es recomendado por profesionales de la salud, tanto en la rehabilitación de personas con enfermedades de base, así como también en la prevención de enfermedades en personas sanas ${ }^{(26)}$. Sin embargo, solo unos pocos ensayos clínicos han investigado la efectividad del Pilates en población sana ${ }^{(27,28)}$ y hasta ahora, por ejemplo, un único meta-análisis ha informado de su efectividad para lograr reducciones significativas en la sintomatología depresiva y ansiosa y en la sensación de fatiga de los pacientes, así como un aumento en su energía ${ }^{(1)}$.

Mokhtari, Nezakatalhossaini y Esfarjani ${ }^{(14)}$ investigaron la eficacia del Pilates en el manejo de sintomatología depresiva y el equilibro postural en población de adultos mayores. Antes de iniciar el programa de Pilates, treinta mujeres adultas mayores (divididas en dos grupos: experimental y control) fueron evaluadas a través de la Escala de Depresión Geriátrica y también a través de pruebas de equilibrio. A continuación, el grupo experimental participó de un programa de Pilates y tras 12 semanas fue nuevamente evaluado. Los resultados del análisis covariante informaron que las participantes del grupo experimental mostraron una disminución de la sintomatología depresiva, el tiempo para la prueba de alcance funcional y las pruebas cronometradas, concluyéndose que el Pilates disminuyó la depresión y mejoró el equilibrio de las mismas, en comparación con el grupo control.

Otro estudio se enfocó en determinar el efecto del Pilates en la salud general de mujeres con diabetes tipo II. En ese estudio, Torabian, Taghadosi, Ajorpaz y Khorasanifar ${ }^{(29)}$ incluyeron a 70 mujeres con diabetes tipo II, las cuales fueron asignadas aleatoriamente en 
dos grupos (experimental y control) de 35 personas cada uno. A todas las participantes se les aplicó el Cuestionario de Salud General de 28 ítems (GHQ28), el cual posee subescalas para medir específicamente sintomatología física, ansiedad, disfunción social y depresión ${ }^{(30,31)}$. Luego, el grupo experimental atravesó un programa de Pilates de 2 sesiones/semana, por 8 semanas, mientras que el grupo control solo recibió medicación. Tras completar el programa, se volvió a evaluar a las pacientes con el GHQ28. Las participantes del grupo experimental mostraron mejorías significativas en la sintomatología física $(p=0,01)$, ansiedad $(p=0,003)$, disfunción social $(p=0,001)$ y depresión $(p=0,04)$, en comparación con el grupo control. Resultados similares también pudieron observarse en el estudio de Yucel y Uysal ${ }^{(32)}$.

Por su parte, las investigaciones de Pourvaghar et al. ${ }^{(33)}$ y de Ashrafinia et al. ${ }^{(34)}$ encontraron que, en comparación con sendos grupos controles, un programa de Pilates mejoraba la depresión, la ansiedad, los trastornos del sueño y síntomas somáticos en varones adultos de edad media y la fatiga física y mental y la motivación en mujeres durante el postparto, respectivamente.

En Turquía, se compararon los efectos del Pilates y del masaje tisular conectivo en la intensidad del dolor, umbral del dolor a la presión, tolerancia, ansiedad, progresión y calidad de vida relacionada con la salud en mujeres con fibromialgia. Veintiún mujeres participaron del grupo Pilates y 22 del grupo de masaje tisular conectivo. En cada grupo, antes y al final del tratamiento, se utilizaron la Escala Analógica Visual, algometría, el Inventario de Rasgo-Estado de Ansiedad, el Cuestionario de Impacto de la Fibromialgia y el Perfil de Salud de Nottingham. Se encontraron mejoras significativas de todos los parámetros medidos, en ambos grupos. Sin embargo, las puntuaciones para el umbral del dolor a la presión fueron significativamente elevadas y los síntomas de ansiedad disminuyeron, también, significativamente en el grupo de Pilates en comparación con el grupo de masaje tisular conectivo ${ }^{(35)}$.

Rahimimoghadam et al. ${ }^{(36)}$ realizaron un estudio controlado en 50 pacientes en hemodiálisis, los cuales fueron aleatoriamente asignados a un grupo experimental y a un grupo control, de 25 personas cada uno. La GHQ28 fue completada por todos los participantes al inicio y al final del tratamiento. El grupo experimental recibió Pilates, 3 veces/semana, durante 8 semanas. Al finalizar el estudio, se evidenció diferencias intragrupo en los pacientes que recibieron Pilates, con mejoras significativas en la puntuación global del GHQ28 $(p=0,002)$ y en todas sus subescalas (síntomas físicos, ansiedad, disfunción social y depresión). Asimismo, las mejorías también eran significativas al comparar los resultados obtenidos entre los grupos experimental y control $(p=0,001)$.

Además de utilizarse en población clínica (adultos mayores, personas con enfermedades crónicas como diabetes o insuficiencia renal, pacientes con fibromialgia, entre otros) como se vio previamente, el Pilates ha demostrado también beneficios físicos y psicológicos en población general sana. Tolnai, Szabo, Koteles y Szabo ${ }^{(37)}$ estudiaron los efectos longitudinales de un programa de Pilates de baja intensidad, 1 vez por semana, durante 10 semanas, en mujeres jóvenes y sanas, aunque sedentarias. En comparación con un grupo control, las mujeres en las que se realizó la intervención exhibieron mejorías significativas en la masa muscular, flexibilidad, balance, fuerza muscular abdominal y de la parte baja de la espalda, conciencia corporal y afectividad positiva, con disminución concomitante de la afectividad negativa. La relativa baja frecuencia del programa ( 1 vez por semana) también pudo verse como una "ventaja" frente a las excusas de falta de tiempo que muchas personas aducen para no realizar ejercicios ${ }^{(37)}$. Además, en población universitaria sana, el Pilates y otros programas de ejercicios basados en el movimiento han demostrado mejorar la autorregulación, la autoeficacia, el estado de ánimo, el afrontamiento del estrés y la calidad del sueño(15).

Akbaş y Ünver ${ }^{(38)}$ investigaron la efectividad de un programa de Pilates sobre parámetros relacionados con la salud de mujeres jóvenes (características antropométricas, estado emocional, fatiga y calidad de vida). Mujeres sedentarias de entre 18 y 25 años fueron asignadas al azar a un grupo de ejercicios de Pilates $(n=25)$ y a un grupo control $(n=26)$. El grupo Pilates realizó un protocolo de ejercicios durante 6 semanas, mientras que el grupo control no realizó ninguna actividad física regular. Las características antropométricas, la ansiedad (medida a través de la Escala de Ansiedad de Beck), la depresión (evaluada a través de la Escala de Depresión de Beck), la fatiga (determinada a través de la Escala de Severidad de la Fatiga) y la calidad de vida (medida a través de la forma corta del 
Cuestionario de Salud de 36 ítems) de las participantes se evaluaron al inicio del estudio y tras seis semanas. Finalizado el estudio, el peso $(p=0,005)$, índice de masa corporal $(p=0,005)$, circunferencia de la cintura $(p<0,001)$, cadera $(p=0,002)$ y muslo $(p<0,001)$, gravedad de la ansiedad $(p=0,001)$, depresión $(p=0,001)$ y fatiga $(p<0,001)$ disminuyeron significativamente en el grupo Pilates. Asimismo, la vitalidad $(p=0,005)$ y los dominios de calidad de vida relacionada a la salud mental $(p=0,021)$ aumentaron. No hubo diferencias significativas en el grupo control $(p>0,005)$, excepto la ansiedad $(p=0,010)$ y la fatiga $(p=0,032)$.

En ninguno de los estudios citados previamente se observaron efectos adversos. Sin embargo, es importante recalcar que la interpretación de los resultados de todas estas investigaciones debe hacerse de manera cautelosa, debido al bajo tamaño muestral de las mismas, a la heterogeneidad metodológica que presentaban, así como a la falta de explicación en detalle del método de Pilates utilizado y a la no uniformidad de las escalas utilizadas para la evaluación psicopatológica de los pacientes. Es por ello que se necesitan más investigaciones, con mayores tamaños de muestra, que permitan comparar los programas de Pilates con otros grupos de intervenciones terapéuticas y que se aseguren de utilizar instrumentos de evaluación psicopatológica validados y útiles, tanto para población clínica como para población general. Todo lo anterior ayudará a asegurar la exactitud en la interpretación de los resultados acerca de la efectividad del Pilates.

El mecanismo por el cual el Pilates mejora la salud mental todavía es objeto de controversia. Algunos investigadores afirman que el Pilates aumenta los niveles de serotonina, con lo que disminuyen los síntomas depresivos ${ }^{(39-41)}$. Otros informan que el mejoramiento de los síntomas podría deberse al aumento de oportunidades de socialización que tienen las personas que practican Pilates ${ }^{(32,35)}$, puesto que la interacción social ha demostrado, por ejemplo, que provoca reducciones significativas en los síntomas depresivos en adultos mayores deprimidos. Asimismo, la disminución de la actividad nerviosa simpática, que se ve debido al principio de respiración profunda que se utiliza en el Pilates, podría ser un mecanismo que explique las mejorías en los síntomas ansiosos y depresivos de algunos pacientes. Esto último es similar a lo que sucede en el Yoga (que también reduce el estrés y la presión arterial a través de un mecanismo de activación parasimpática), que ha tenido evidencia positiva para el manejo de la ansiedad que acompaña a varios trastornos mentales ${ }^{(42)}$. Además, el control de grandes grupos musculares en coordinación y el hecho de enfocarse en la respiración pueden aumentar la capacidad aeróbica del individuo y mejorar así la salud mental ${ }^{(15,43)}$.

Es importante destacar que algunos estudios no han sido capaces de demostrar efectos positivos del Pilates sobre las variables psicológicas ${ }^{(44)}$, aunque tampoco han informado de efectos adversos. Lo anterior es importante a la hora de tomar decisiones terapéuticas por parte del médico psiquiatra y del fisioterapeuta.

\section{CONCLUSIONES}

Los resultados de esta revisión sugieren que existe evidencia preliminar que apoya que el Pilates puede mejorar la salud mental y las variables psicológicas de las personas, sin importar su estado de salud, con importante potencial para reducir síntomas de depresión y ansiedad. Lo anterior está en consonancia con algunas revisiones sistemáticas realizadas sobre los efectos de la actividad física y el bienestar en adultos mayores ${ }^{(45-49)}$, y sobre síntomas de ansiedad en los pacientes ${ }^{(5)}$. Asimismo, la evidencia disponible apoya que la participación en este tipo de programas físicos puede ser importante en la facilitación y el manejo de síntomas psicológicos asociados a enfermedades crónicas (por ejemplo, diabetes mellitus, enfermedad renal crónica), así como también en personas sanas sedentarias.

Entonces, ¿sirve el Pilates para mejorar la salud mental? En corto, y aunque la evidencia todavía es limitada, se puede afirmar que sí. El Pilates puede adaptarse a las necesidades físicas particulares de cada individuo y su ejecución y progresión se basan en la capacidad individual del mismo ${ }^{(50)}$. Al no haberse reportado efectos adversos en las investigaciones, se sugiere que el Pilates es potencialmente seguro y eficaz para mejorar la salud mental en todas las edades. 
Se necesita más investigación, bien diseñada, con grandes muestras de adultos, hombres y mujeres jóvenes, sin patologías de base, para determinar si el Pilates puede generar mejoras significativas en los resultados de salud mental en individuos por lo demás sanos. También se necesitan ensayos controlados aleatorizados rigurosos que permitan examinar los efectos del Pilates en pacientes con enfermedades crónicas asociadas con un mayor riesgo de ansiedad, síntomas depresivos y otros estados psicopatológicos.

\section{REFERENCIAS BIBLIOGRÁFICAS}

1. Fleming KM, Herring MP. The effects of Pilates on mental health outcomes: A meta-analysis of controlled trials. Complement Ther Med. 2018;37:80-95.

2. Mikkelsen K, Stojanovska L, Polenakovic M, Bosevski M, Apostopoulos V. Exercise and mental health. Maturitas. 2017;106:48-56.

3. Torales J, Barrios I, Almirón M, De la Cueva R. Physiotherapy in the treatment of anxiety disorders. International Journal of Culture and Mental Health 2017;10(3): 298-99.

4. Conn VS. Anxiety outcomes after physical activity interventions: meta-analysis findings. Nurs Res. 2010;59(3):224-31.

5. Herring MP, O'Connor PJ, Dishman RK. The effect of exercise training on anxiety symptoms among patients: a systematic review. Arch Intern Med. 2010;170(4):321-31.

6. Herring MP, Puetz TW, O'Connor PJ, Dishman RK. Effect of exercise training on depressive symptoms among patients with a chronic illness: a systematic review and meta-analysis of randomized controlled trials. Arch Intern Med. 2012;172:101111. Arch Intern Med. 2012;172(2):101111. rep

7. Schuch FB, Deslandes AC, Stubbs B, Gosmann NP, da Silva CTB, de Almeida Fleck MP. Neurobiological effects of exercise on major depressive disorder: a systematic review. Neurosci Biobehav Rev. 2016;61:1-11.

8. Torales J, Almirón M, González I, Gómez N, Ortiz R, Ibarra V. Ejercicio físico como tratamiento adyuvante de los trastornos mentales. Una revisión narrativa. An. Fac. Cienc. Méd. (Asunción) 2018; 51(3):27-32.

9. Ortiz R, Ibarra V, Almirón M, González I, Gómez N, Torales J. Fisioterapia en Salud Mental: una breve guía introductoria. $1^{a}$ ed. Asunción: EFACIM; 2018.

10. Pilates JH. Your Health. New York, NY: Presentation Dynamics Incorporated; 1934.

11. Wells C, Kolt GS, Bialocerkowski A. Defining Pilates exercise: a systematic review. Complement Ther Med. 2012;20(4):253-62.

12. Cruz-Ferreira A, Fernandes J, Laranjo L, Bernardo LM, Silva A. A systematic review of the effects of Pilates method of exercise in healthy people. Arch Phys Med Rehabil. 2011;92(12):2071-81.

13. Lim ECW, Poh RLC, Low AY, Wong WP. Effects of Pilates-based exercises on pain and disability in individuals with persistent nonspecific low back pain: a systematic review with meta-analysis. J Orthop Sports Phys Ther. 2011;41(2):70-80.

14. Mokhtari M, Nezakatalhossaini M, Esfarjani $F$. The effect of 12-week Pilates exercises on depression and balance associated with falling in the elderly. Procedia Soc Behav Sci. 2013;70:1714-23.

15. Caldwell K, Harrison M, Adams M, Quin RH, Greeson J. Developing mindfulness in college students through movement based courses: effects of self-regulatory self efficacy, mood, stress and sleep quality. J Am Coll Health. 2010;58(5):433-42.

16. Garcia-Soidan JL, Arufe Giraldez V, Cachon Zagalaz J, Lara-Sanchez AJ. Does Pilates exercise increase physical activity, quality of life, latency, and sleep quantity in middle-aged people? Percept Mot Skills. 2014;119(3):838-50.

17. de Siqueira Rodrigues BG, Cader SA, Torres NVOB, de Oliveira EM, Dantas EHM. Pilates method in personal autonomy, static balance and quality of life of elderly females. J Bodyw Mov Ther. 2010;14(2):195-202.

18. Akbas E, Erdem EU. Does Pilates-based approach provide additional benefit over traditional physiotherapy in the management of rotator cuff tendinopathy? A randomised controlled trial. Ann Sports Med Res 2016;3(6): 1083.

19. Karaman A, Yuksel I, Kinikli GI, Caglar O. Do Pilates-based exercises following total knee arthroplasty improve postural control and quality of life? Physiother Theory Pract 2017; 33(4):289-95.

20. Campos de Oliveira L, Gonçalves de Oliveira R, Pires-Oliveira DAdA. Effects of Pilates on muscle strength, postural balance and quality of life of older adults: a randomized, controlled, clinical trial. J Phys Ther Sci. 2015; 27(3): 871-76.

21. Frank R, Edwards K, Larimore J. Yoga and Pilates as Methods of Symptom Management in Multiple Sclerosis. In: Watson R, Killgore WDS, editors. Nutrition and Lifestyle in Neurological Autoimmune Diseases: Multiple Sclerosis. Massachusetts: Academic Press; 2017. p. 189-94.

22. Ginsberg RL, Tinker L, Liu J, Gray J, SangiHaghpeykar H, Manson JE et al. Prevalence and correlates of body image dissatisfaction in postmenopausal women. Women Health 2016;56(1):23-47. 
23. Rogers K, Gibson AL. Eight-week traditional mat Pilates training-program effects on adult fitness characteristics. Res Q Exerc Sport 2009;80(3):569-74.

24. Roh SY. Effect of a 16-week Pilates exercise program on the ego resiliency and depression in elderly women. J Exerc Rehabil 2016;12(5):494-98.

25. Soysal Tomruk M, Uz MZ, Kara B, İdiman E. Effects of Pilates exercises on sensory interaction, postural control and fatigue in patients with multiple sclerosis. Mult Scler Relat Disord. 2016;7:70-3.

26. Herrera-Gutiérrez $E$, Olmos-Soria $M$, Brocal-Pérez D. Efectos psicológicos de la práctica del Método Pilates en una muestra universitaria. Anal. Psicol. 2015 31(3): 916-20.

27. Bavli $\mathrm{O}$, Koybasi $\mathrm{O}$. Investigation the effects of 6 weeks Pilates exercises on biomotorical variables and self-esteem scores of young women. Turkish Journal of Sport and Exercise 2016;18(1):127-31.

28. Parikh CM, Arora M. Role of Pilates in rehabilitation: A literature review. International Journal of Therapies and Rehabilitation Research 2016;5(4):77-83.

29. Torabian $M$, Taghadosi M, Ajorpaz NM, Khorasanifar L. The effect of Pilates exercises on general health in women with type 2 diabetes. J Life Sci. 2013;10:28388.

30. Goldberg DP, Blackwell B. Psychiatric illness in general practice: a detailed study using a new method of case identification. Br Med J. 1970; 2(5707):439-43.

31. Homman A. Standardization and normalization of GHQ-28 [dissertation]. Psychology Faculty: Tarbiate Modares University; 1998.

32. Yucel $H$, Uysal $O$. Pilates-based mat exercises and parameters of quality of life in women with Type 2 diabetes. Iran Red Crescent Med J. 2016;18(3):e21919.

33. Pourvaghar MJ, Bahram ME, Sharif MR, Sayyah M. Effects of eight weeks of Pilates exercise on general health condition of aged male adults. IJSS. 2014;4:895-900.

34. Ashrafinia F, Mirmohammadali M, Rajabi $H$, Kazemnejad A, Sadeghniiat Haghighi K, Amelvalizadeh M. Effect of Pilates exercises on postpartum maternal fatigue. Singapore Med J. 2015;56(3):169-73.

35. Ekici G, Unal E, Akbayrak T, Vardar-Yagli $N$, Yakut $Y$, Karabulut $E$. Effects of active/passive interventions on pain, anxiety, and quality of life in women with fibromyalgia: randomized controlled pilot trial. Women Health. 2017;57(1):88-107.

36. Rahimimoghadam Z, Rahemi Z, Ajorpaz $N M$, Sadat Z. Effects of Pilates exercise on general health of hemodialysis patients. J Bodyw Mov Ther. 2017;21(1)86-92.

37. Tolnai N, Szabo Z, Koteles F, Szabo A. Physical and psychological benefits of once-aweek Pilates exercises in young sedentary women: a 10-week longitudinal study. Physiol Behav. 2016;163:211-18. Doi: $10.1016 /$ j.physbeh.2016.05.025

38. Akbas $E$, Ünver B. A six-week Pilates exercise protocol for improving physical and mental health-related parameters. Malaysian Journal of Movement, Health \& Exercise 2018;7(2):65-79.

39. Hassan EAH, Amin MA. Pilates exercises influence on the serotonin hormone, some physical variables and the depression degree in battered women. World J. Sport Sci. 2011;5(2):89-100.

40. Nicoloff G, Schwenk TL. Using Exercise to Ward Off Depression. Phys Sportsmed.1995;23(9):44-58.

41. Delgado PL, Moreno FA. Role of norepinephrine in depression. J Clin Psychiatry. 2000;61(Suppl 1):5-12.

42. Torales J, Barrios I, Villalba J. Alternative Therapies for Excoriation (Skin Picking) Disorder: A Brief Update. Adv Mind Body Med. 2017;37(1):10-3.

43. Kucuk F, Livanelioglu A. Impact of the clinical Pilates exercises and verbal education on exercise beliefs and psychosocial factors in healthy women. J Phys Ther Sci 2015;27(11):3437-43. Doi: 10.1589/jpts.27.3437

44. Cruz-Ferreira A, Fernandes J, Gomes D, Bernardo LM, Kirkcaldy BD, Barbosa TM, et al. Effects of Pilates-based exercise on life satisfaction, physical self-concept and health status adult women. Women Health 2011;51(3):240-55.

45. Bullo V, Bergamin M, Gobbo S, Sieverdes JC, Zaccaria M, Neunhaeuserer D, et al. The effects of Pilates exercise training on physical fitness and wellbeing in the elderly: a systematic review for future prescription. Prev Med. 2015;75:1-11.

46. Chodzko-Zajko WJ, Proctor DN, Fiatarone Singh MA, Minson CT, Nigg CR, Salem GJ, et al. American College of Sports Medicine position stand. Exercise and physical activity for older adults. Med Sci Sports Exerc. 2009;41(7):1510-30.

47. Cruice M, Worrall L, Hickson L. Reporting on psychological well-being of older adults with chronic aphasia in the context of unaffected peers. Disabil Rehabil. 2011;33(3):219-28.

48. Irez GB, Ozdemir RA, Evin R, Irez SG, Korkusuz $\mathrm{F}$. Integrating Pilates exercise into an exercise program for $65+$ year-old women to reduce falls. J Sports Sci Med. 2011;10(1):105-11.

49. Keysor J]. Does late-life physical activity or exercise prevent or minimize disablement? A critical review of the scientific evidence. Am J Prev Med. 2003; 25(3 Suppl 2):12936.

50. McNeill W. Decision making in Pilates. J Bodyw Mov Ther. 2011;15(1):103-7. 